\title{
INTERVENÇÕES DE ENFERMAGEM NA PREVENÇÃO DA PAVMI NO SERVIÇO DE URGÊNCIA
}

\author{
NURSING INTERVENTIONS IN VAP PREVENTION IN THE EMERGENCY \\ DEPARTMENT \\ INTERVENCIONES DE ENFERMERÍA EN LA PREVENCIÓN DE LA NAV EN \\ URGENCIAS
}

Paulo Jorge Rodrigues Sabino ${ }^{1}$

RESUMO: Enquadramento: A implementação de medidas preventivas na prevenção da Pneumonia Associada à Ventilação Mecânica Invasiva (PAVMI) deve considerar-se uma prioridade para os enfermeiros, pela importância que estes assumem no planeamento e concretização das mesmas, visando a redução da incidência da PAVMI tardia. Objetivo: analisar a evidência científica existente sobre a importância do enfermeiro, identificando as suas intervenções na prevenção da PAVMI em contexto de serviço de urgência. Metodologia: revisão sistemática da literatura, com análise de artigos publicados entre 2018 e 202I nas bases PubMed, B-ON e EBSCO, com descritores MeSH e DeCS, selecionando 3 artigos. Resultados: foram obtidas uma série de intervenções nas quais o enfermeiro é o responsável pelo seu planeamento, implementação, execução e avaliação, como a aspiração de secreções com técnica assética ou a higiene oral com antisséticos tópicos como a clorohexidina a o,2\%. Conclusão: o enfermeiro é o profissional indicado para o planeamento e implementação de medidas de prevenção efetivas na redução da infeção por PAVMI, em virtude das suas competências profissionais e pessoais. Acresce-se ainda ao enfermeiro do Serviço de Urgência a necessidade precoce da implementação destas intervenções, reconhecendo-se o seu papel fulcral na prevenção e/ou minimização dos efeitos tardios relacionados com a PAVMI.

Palavras chave: Pneumonia. infeção. Cuidados de enfermagem. Ventilação mecânica.

ABSTRACT: Background: the implementation of preventive measures for the prevention of Ventilator Associated Pneumonia should be considered a priority for nurses, due to their importance in the planning and implementation of these measures, looking in reducing the incidence of late VAP. Objective: analyze the existing scientific evidence on the importance of nurses, identifying their interventions in preventing VAP in the emergency department. Methodology: systematic literature review, with analysis of articles published between 2018 and 2021 in PubMed, B-ON and EBSCO, with MeSH and DeCS descriptors, selecting 3 articles. Results: a set of interventions were obtained in

\footnotetext{
${ }^{1}$ Mestrado em Enfermagem - pela Universidade de Évora. Enfermeiro de Cuidados Gerais na Medicina II Hospital José Joaquim Fernandes - ULSBA, EPE, Beja, Portugal. ORCID: https://orcid.org/oooo-ooo3-23293072
} 
which the nurse is responsible for their planning, implementation, execution and evaluation, such as aspiration of secretions with aseptic technique or oral hygiene with topical antiseptics such as $0.2 \%$ chlorohexidine. Conclusion: nurses are the recommended professionals to plan and implement effective prevention measures to reduce VAP infection, due to their professional and personal skills. In addition, emergency department nurses need to implement these interventions, from a early basis, recognizing their key role in preventing and/or minimizing the VAP late effects.

Keywords: Pneumonia. infection. Nursing care. Mechanical ventilation.

RESUMEN: Antecedentes: la implementación de medidas preventivas para la prevención de la Neumonía Asociada a la Ventilación debe ser considerada una prioridad para el personal de enfermería, debido a su importancia en la planificación e implementación de estas medidas, con el fin de reducir la incidencia de la NAV tardía. Objetivo: analizar la evidencia científica existente sobre la importancia del enfermero, identificando sus intervenciones en la prevención de la NAV en urgencias. Metodo: revisión bibliográfica sistemática, con análisis de artículos publicados entre 2018 y 2021 en PubMed, B-ON y EBSCO, con descriptores $\mathrm{MeSH}$ y DeCS, seleccionando 3 artículos. Resultados: se obtuvieron una serie de intervenciones en las que los enfermeros son los responsables por su planificación, implementación, ejecución y evaluación, como la aspiración de secreciones con técnica aséptica o la higiene bucal con antisépticos tópicos como la clorohexidina al o,2\%. Conclusiones: Los enfermeros son los profesionales adecuados para planificar y aplicar medidas de prevención eficaces para reducir la infección por NAV, debido a sus competencias profesionales y personales. El personal de enfermería de los servicios de urgencias también debe aplicar estas intervenciones en una fase temprana, reconociendo su papel clave en la prevención y minimización de los efectos tardíos relacionados con la NAV.

Palabras-clave: Neumonía. Infección. Atención de enfermería. Ventilación mecánica.

\section{INTRODUÇÃO}

Uma das infeções mais frequentes relacionadas com cuidados de saúde é a Pneumonia Associada à Ventilação Mecânica Invasiva (PAVMI), com intervalos significativos de incidência, valores muito relevantes de mortalidade e morbilidade e com elevados custos associados, sendo Portugal um dos países a nível europeu com maior taxa de infeção, com valores estimados de 7,8\%, de acordo com o último estudo epidemiológico realizado em 2017 (Direção-Geral da Saúde [DGS], 2017a).

A PAVMI, sendo uma Infeção Associada aos Cuidados de Saúde (IACS) e, sabendo que a larga maioria destas é evitável através de medidas eficazes de prevenção e controlo de infeção (Rodrigues, 2019), deve ser combatida, primeiramente através da prevenção e controlo, através de boas práticas clínicas e intervenções direcionadas e só 
depois, caso a prevenção falhe, através da implementação racional de terapêutica direcionada à mesma (Pina et al., 2010).

Assim, é neste sentido que as intervenções de enfermagem assumem especial importância, uma vez que a larga maioria das intervenções preconizadas na prevenção da PAVMI é realizada por enfermeiros, tornando-os assim nos profissionais mais vocacionados para a implementação e execução das medidas preconizadas para a sua prevenção, muito devido às suas competências profissionais e pessoais, o que, a médio e longo prazo, vai permitir a redução das IACS, a redução dos tempos de internamento e a redução de custos hospitalares. Nesta medida, procura-se analisar a evidência científica existente sobre a importância do enfermeiro, identificando as suas intervenções na prevenção da PAVMI em contexto de serviço de urgência.

\section{ENQUADRAMENTO / FUNDAMENTAÇÃO TEÓRICA:}

As IACS são "uma infeção adquirida pelos doentes em consequência dos cuidados e procedimentos de saúde prestados" (Ordem dos Enfermeiros [OE], 2017, p. II), englobando, de forma obvia, todas as unidades que prestam cuidados de saúde. São ainda referidos como "uma condição sistémica ou localizada (...) à presença de um agente infecioso, que não estava presente ou em incubação no momento da admissão na unidade hospitalar" (WHO, 20II, citado por Simões, 2019, p. 6) mas sem ser restrito a estas, como tínhamos visto já anteriormente, podendo afetar tanto doentes, como profissionais de saúde (Simões, 2016). De origem variada e multifatorial (Lobão et al., 2016; Pina et al., 20ı; Simões, 2016), incluem, segundo Simões (2016, p. 16, referindo Ducel et al., 2002), "fatores relacionados com o agente microbiano, suscetibilidade do indivíduo, fatores ambientais e resistência bacteriana", destacando-se os terceiros, os fatores ambientais, onde se incluem as transferências inter-serviços e a sobrelotação dos hospitais, com aglomeração excessiva de pessoas num espaço reduzido e ocorrem, essencialmente, devido ao forte avanço tecnológico ocorrido transversalmente em todas as áreas, inclusive na área da saúde, sobretudo nos últimos anos, com desenvolvimento de novas tecnologias, com repercussão evidente em cuidados de saúde mais eficazes, sempre com a perspetiva de aumentar o ciclo vital, o que levou a um envelhecimento da população, com reflexo no "aumento significativo de doenças crónicas e (...) elevado número de pessoas portadoras de múltiplas 
patologias que exigem uma complexidade de cuidados inquestionável” (Ministério da Saúde, 2018, p. 7), com a consequente necessidade da implementação e utilização de novos dispositivos médicos e meios de diagnóstico e tratamento (Lobão et al., 2016).

Atualmente, as IACS constituem um sério problema de saúde devido aos elevados custos associados e à elevada mortalidade e morbilidade (DGS, 2017a; Lobão et al., 2016; Pina et al., 2010; Reis, 2017, referindo Bonten, 2011, ECDC, 2012 e Matos \& Sobral, 2010; Simões, 2016, referindo Costa et al., 2009, Ribeiro, 201r e WHO, 2009), já para não falar da ameaça que constitui à segurança dos doentes (Rodrigues, 2019), embora também se refira que uma em cada três infeções possa ser evitada (Simões, 2016, referindo Ribeiro, 20Ir e Silva, 2008), através "de medidas efetivas de prevenção e controlo de infeção" (Rodrigues, 2019, p. 25, referindo CDC, 2016a, OECD, 2016 e WHO, 2016). Estima-se que, de acordo com vários estudos internacionais, incluindo um estudo realizado pelo European Centre for Disease Prevention and Control (ECDC), para os anos de 2011 e 2012, a incidência das IACS tenha afetado, sensivelmente, cerca de 3 milhões de pessoas por ano (Pina et al., 2010; Simões, 2016), sendo um claro "um indicador da qualidade dos cuidados prestados aos doentes nas unidades de saúde" (Lito, 2010, p. 25). Relativamente a Portugal, tem-se verificado uma evolução lenta mas significativa, no sentido decrescente das taxas de incidências das principais IACS, com destaque para uma descida de cerca de $36 \%$ na Pneumonia Associada ao Ventilador (PAV) no período entre 2008 e 2016 (Lobão et al., 2016).

A PAVMI é referida como uma das três formas de pneumonia (Jameson et al., 2015)(tradução livre), surge na sequência da utilização da VMI, com incidências até 52\% dos doentes com necessidade de VMI (idem) e pode ser classificada de precoce ou tardia, consoante ocorra, respetivamente, até às 96 horas após internamento ou após as $96 \mathrm{~h}$, com pelo menos 48 horas de VMI (Ferreira et al., 2017; Frota et al., 2019). Decorre da colonização por microrganismos epidemiologicamente significativos, como a Klebsiella spp, o Staphylococcus aureus e a Pseudomonas aeruginosa (Ferreira et al., 2017, referindo Batista et al., 2013), microrganismos com caraterísticas de multirresistência (Ferreira et al., 2017), podendo esta colonização ocorrer em qualquer porção das vias aéreas (superiores ou inferiores) mas também ao nível da cavidade oral, uma vez que a redução do nível de consciência induzido pela medicação associada à manutenção de dispositivos médicos 
(curarização, sedação e/ou analgesia) leva a uma diminuição ou ausência de higiene oral por parte da pessoa doente (Ferreira et al., 2017). Refere-se ainda que a presença do TET promove a "diminuição significativa do mecanismo de defesa pulmonar" (Reis, 2017, p. I7, referindo ECDC, 2012 e Perkins et al., 2010), podendo originar "tosse ineficaz, disfunção mucociliar e numa alteração do adequado encerramento da epiglote, ocasionando microaspirações" (Reis, 2017, p. 26), tornando-se assim num fator de risco de desenvolvimento de infeções, ao mesmo tempo que se considera um dispositivo de último recurso. Os fatores de risco de ocorrência da PAVMI residem no enfraquecimento do sistema imunitário da pessoa ventilada, o que leva, para além de uma maior probabilidade de contaminação das vias aéreas, à presença de microrganismos multirresistentes no meio ambiente, à utilização de antibioterapia de largo espectro por longos períodos e ao uso prolongado do ventilador (Frota et al., 2019). As medidas de prevenção preconizadas na prevenção da PAVMI consistem num conjunto de intervenções, conhecidas como bundles, os quais são considerados ferramentas de "all or nothing", uma vez que se considera que o conjunto, razão pela qual é referido, deve ser implementado na sua totalidade, sem aplicação de intervenções isoladas, sob pena de não surtirem efeito (DGS, 2oiłb). Estas, assumem um caráter multidisciplinar, devendo apenas ser executadas por profissionais de saúde capacitados, nos quais se inserem os enfermeiros, os quais, pelas suas competências, dentro da sua autonomia profissional e respaldados por normativos legais (Decreto-Lei no 161/96, de 4 de setembro), assumem o planeamento, implementação, execução e avaliação da larga maioria das intervenções, tornando-se assim os profissionais de referência na prevenção da PAVMI.

Atualmente, para Portugal, o estado da arte remete para uma norma emitida pela DGS, a norma no 21/2012, de 16 de dezembro, com atualização a 30 de maio de 2017 (DGS, $2017 b)$, na qual se podem encontrar as intervenções previstas para a prevenção associada à intubação (PAI), não existindo uma norma específica para a prevenção da PAVMI, muito embora o conteúdo desta norma, em termos técnicos e funcionais, possa ser eficazmente adaptado à PAVMI. Esta engloba seis intervenções major, com indiferenciação profissional, sendo as mesmas (DGS, 2017b):

- A revisão, redução ou suspensão diária, ou definitiva, da sedação do doente com VMI; 
- A discussão e avaliação diária da realização de desmame ventilatório e/ou extubação do doente;

- A manutenção da cabeceira da cama num ângulo entre 30 e $45^{\circ}$;

- A realização, no mínimo três vezes diárias, de higiene oral do doente com antisséticos orais, recorrendo à clorohexidina $0.2 \%$;

- A manutenção de circuitos ventilatórios limpos, efetuando a troca se visivelmente sujos;

- A manutenção da pressão do cuff (ou balão) do TET entre 20 e $30 \mathrm{mH}_{2} \mathrm{O}$.

\section{PROCEDIMENTOS METODOLÓGICOS DE REVISÃO}

Foi realizada uma revisão sistemática da literatura existente, pela forma como a mesma "procura sintetizar e resumir o conhecimento existente em vez de criar novo conhecimento" (Aromataris \& Pearson, 2014, citado por Santos et al., 2018, p.2), levando a que a tomada de decisão seja de acordo com a melhor evidência disponível no momento, tendo em conta a eficácia e a pertinência da prática a desenvolver (Santos et al., 2018), já que os "profissionais de saúde precisam de provas para fundamentar (...) atividades e intervenções (Institute TJB, 2014 e Pearson, Wiechula, Court \& Lockwood, 2005, citados por Santos et al., 2018, p.I).

Recorreu-se à metodologia PRISMA da Joanna Briggs Institute (Aromataris \& Munn, 2020), com o objetivo de analisar a evidência científica existente sobre a importância do enfermeiro e das suas intervenções no âmbito da prevenção da PAVMI no serviço de urgência. A pesquisa foi realizada entre maio e junho de 2021, nas bases de dados PubMed, B-ON e EBSCOhost, com friso cronológico entre 2018 e 202I, com recurso a descritores $\mathrm{MeSH}$ e DeCS, tendo sido considerados todos os artigos com abstract, decorrentes da conjugação das palavras "ventilation" (apenas no título), "mechanical”, "pneumonia”, "nursing”, estas com utilização do booleano AND, conjuntamente com "intensive care", tendo esta o booleano NOT, assumindo a forma "(ventilation [Title]) AND (mechanical) AND (pneumonia) AND (nursing) NOT (intensive care)". Assumiram-se como critérios de exclusão, para além de artigos com referência aos cuidados intensivos (referido no booleano NOT), artigos que não possuíssem abstract ou que referissem crianças ou COVID-ı.

Após a aplicação das várias fases da metodologia, obtiveram-se 188 artigos, tendo sido tendo sido selecionados 3 , sendo 2 da base B-ON e I da base EBSCOhost (figura I). 
Figura I - Diagrama PRISMA FLOW

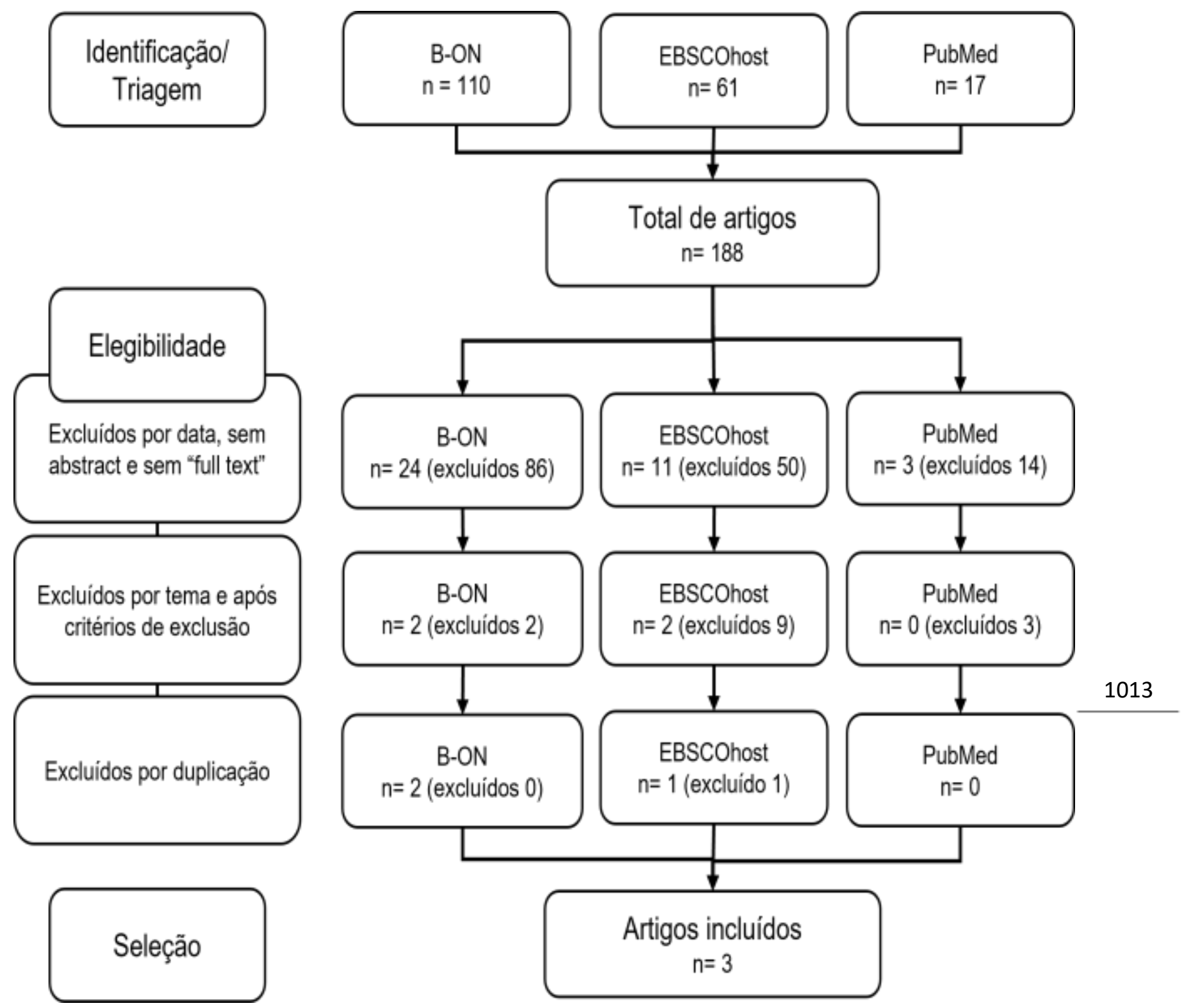

Fonte: do autor

\section{RESULTADOS}

Após a seleção dos artigos, os mesmos foram submetidos à avaliação crítica, através de grelhas de avaliação, seguindo as ferramentas da JBI em termos de evidência, confiabilidade e relevância e as normas da Effective Public Health Practice Project (EPHPP) em termos da avaliação da qualidade metodológica e recomendação (tabela I). 
Tabela I - Avaliação crítica de artigos (evidência, confiabilidade, relevância e recomendação)

\begin{tabular}{|c|c|c|c|c|}
\hline Autores & Título & Objetivo & Análise & País/Ano \\
\hline $\begin{array}{l}\text { Maran, E., Melo, } \\
\text { W., Spigolon, } \\
\text { D., Teston, E., } \\
\text { Tostes, M. \& } \\
\text { Barreto, M. }\end{array}$ & $\begin{array}{l}\text { Prevenção da } \\
\text { pneumonia } \\
\text { associada à } \\
\text { ventilação } \\
\text { mecânica sob } \\
\text { a ótica de } \\
\text { académicos de } \\
\text { enfermagem }\end{array}$ & $\begin{array}{c}\text { Descrever os } \\
\text { conhecimentos dos } \\
\text { alunos de } \\
\text { enfermagem dos anos } \\
2013 \text { a } 2016 \text { sobre a } \\
\text { prevenção da } \\
\text { Pneumonia Associada } \\
\text { à Ventilação Mecânica }\end{array}$ & $\begin{array}{l}\text { Estudo de caso, qualitativo } \\
\text { Relevância e confiabilidade } \\
\text { de } 100 \% \\
\text { Nível de evidência JBI: } 4 \\
\text { Recomendação: baixa }\end{array}$ & $\begin{array}{c}\text { Brasil, } \\
2019\end{array}$ \\
\hline $\begin{array}{l}\text { Oliveira, A., Rocha, } \\
\text { A., Silva, C., } \\
\text { Matos, D., Lima, } \\
\text { E., Anjos, E., } \\
\text { Silva, F., Silva, I., } \\
\text { Mendes, J., } \\
\text { Nogueira, P., Silva, } \\
\text { R., Luz, S., Lopes, } \\
\text { T., Guimarães, V., } \\
\text { Silva, W. \& } \\
\text { Nascimento, W. }\end{array}$ & $\begin{array}{l}\text { Pneumonia } \\
\text { Associada à } \\
\text { Ventilação } \\
\text { Mecânica: o } \\
\text { cuidar da } \\
\text { enfermagem }\end{array}$ & $\begin{array}{l}\text { Descrever os } \\
\text { principais cuidados } \\
\text { realizados por } \\
\text { enfermeiros na } \\
\text { prevenção da PAVMI }\end{array}$ & $\begin{array}{l}\text { Revisão integrativa. } \\
\text { Relevância e confiabilidade } \\
\text { de } 63,6 \% \\
\text { Nivel de evidência JBI: } 2 \text { a } \\
\text { Recomendação: moderada }\end{array}$ & $\begin{array}{c}\text { Brasil, } \\
2019\end{array}$ \\
\hline $\begin{array}{c}\text { Xu, Y., Lai, C., } \\
\text { Xu, G., Meng, } \\
\text { W., Zhang, J., } \\
\text { Hou, H. \& Pi, H. }\end{array}$ & $\begin{array}{l}\text { Risk factors of } \\
\text { ventilator } \\
\text { associated } \\
\text { pneumonia in } \\
\text { elderly } \\
\text { patients } \\
\text { receiving } \\
\text { mechanical } \\
\text { ventilation }\end{array}$ & $\begin{array}{l}\text { Verificar os potenciais } \\
\text { fatores de } \\
\text { risco associados à } \\
\text { pneumonia } \\
\text { associada à ventilação } \\
\text { mecânica } \\
\text { em doentes com } \\
\text { Eẹnteç̧ø autor } \\
\text { mecânica }\end{array}$ & $\begin{array}{l}\text { Estudo transversal, } \\
\text { retrospetivo } \\
\text { Relevância e confiabilidade } \\
\text { de } 75 \% \\
\text { Nivel de evidència JBI: } 3 b \\
\text { Recomendação: moderada }\end{array}$ & $\begin{array}{c}\text { China, } \\
2019\end{array}$ \\
\hline
\end{tabular}

Dos artigos selecionados, obtiveram-se um conjunto de intervenções, essenciais para a prevenção e mitigação da PAVMI, em que o enfermeiro é o único responsável pelo seu planeamento, implementação, execução e avaliação. É referida ainda a necessidade de capacitação dos profissionais de saúde, nomeadamente os enfermeiros, na identificação, interpretação e atuação adequada no doente com VMI (Maran et al., 2org; Oliveira et al., 2020), bem como na aquisição e desenvolvimento de competências técnicas e práticas para reduzir complicações e custos.

Estas intervenções, implementadas em conjuntos, são também denominadas de "bundles" (Maran et al., 2019) e incluem as seguintes intervenções: 
- A higienização das mãos é uma das principais medidas de prevenção de infeções cruzadas, já que as mãos são consideradas como o veículo primordial de disseminação de infeções (Maran et al., 2019);

- A colocação de uma SNG, para além de promover aporte calórico e proteico ao doente, permite avaliar e prevenir a ocorrência de refluxo gástrico para as vias aéreas (Maran et al., 2019; Oliveira et al., 2020);

- A manutenção da cabeceira da cama entre os 30 e os $45^{\circ}$, o que vai permitir uma "melhoria no volume corrente ventilatório" (Maran et al., 2019, p. 121), com redução dos casos de atelectasias, contribuindo ainda para a prevenção da broncoaspiração (Maran et al., 2019);

- A manutenção de pressão do cuff do TET se situe entre os 20 e $30 c \mathrm{mH}_{2} \mathrm{O}$, o que previne lesões endotraqueais e evita que as secreções da região subglótica alcancem o pulmão (Maran et al., 2019);

- A manutenção dos circuitos respiratórios limpos, com troca de componentes nunca antes das 48 horas de utilização, devendo ser o mais reduzida possível, uma vez que "quanto menor o intervalo das trocas, maior a probabilidade de contaminação" (Maran et al., 2019, p. 121);

- A realização da higiene oral com recurso a antisséticos tópicos, como o gluconato de clorohexidina a $0,12 \%$ ou clorohexidina a 0,2\%, no mínimo três a quatro vezes diárias (Maran et al., 2019), permite a redução da colonização da cavidade oral, prevenindo a halitose e mantendo um nível reduzido de secreções na cavidade oral (Oliveira et al., 2020), esta última com necessidade de recurso à aspiração de secreções;

- A aspiração de secreções, quando efetuada de forma adequada, contribui decisivamente para a prevenção da PAVMI (Maran et al., 2019; Oliveira et al., 2020), devendo ser realizado de forma assética (idem).

Para além destas intervenções, as quais são da exclusiva responsabilidade do enfermeiro, obtiveram-se duas intervenções, de caráter interdependente, uma vez que dependem de outras classes profissionais. São referidas:

- A avaliação diária da sedação dos doente com VMI permite a avaliação da capacidade de manutenção de ventilação espontânea e avaliação do padrão respiratório do doente, como medida prévia à extubação do mesmo, possibilitando a redução do tempo de VMI, diminuindo a incidência da PAVMI (Maran et al., 2019; Oliveira et al., 2020);

- A necessidade de redução de antibioterapia profilática, uma vez que a administração indiscriminada de antibióticos, 
frequentemente em dose dupla ou tripla, leva ao aumento da resistência microbiana à medicação mas também à diminuição das defesas do organismo, com o consequente aumento do risco de infeções por agentes oportunistas, existindo posteriormente a necessidade de instituição de nova antibioterapia direcionada ou antibioterapia com maior poder de penetração nos microrganismos (Xu et al., 2019).

Conclui-se então que, com a implementação de todas as intervenções acima referidas, preferencialmente em "bundles", para além da necessidade de reforço de conhecimentos, técnicos, teóricos e práticos, por parte dos profissionais de saúde, é ainda realçada a importância da necessidade de manutenção das boas práticas por parte dos profissionais (Maran et al., 2019; Oliveira et al., 2020), sendo este um fator transversal a todos os artigos incluídos, uma vez que foram detetadas falhas ao nível de manutenção das mesmas (Maran et al., 2019, Oliveira et al., 2020; Xu et al., 2019), salientando-se ainda a importância dos enfermeiros para a prevenção da PAVMI, uma vez que as intervenções referidas encontram-se na esfera da responsabilidade profissional destes (Oliveira et al., 2020).

Em sentido inverso, é também referido que uma das maiores dificuldades na aplicação dos bundles reside nos profissionais, os quais se mostram renitentes em alterar práticas já instituídas e/ou realizadas por si e sobre as quais as consideram as mais atuais, sem qualquer tipo de evidência científica atual, o que implicaria uma mudança de atitude e comportamentos, fato que, se revertido, poderá levar à melhoria dos índices de incidência e prevalência da PAVMI (Maran et al., 2019; Oliveira et al., 2020; Xu et al., 2019).

\section{DISCUSSÃO}

Verifica-se que as intervenções obtidas através dos estudos selecionados, comparativamente com o estado de arte, existe uma consonância quase completa em termos de intervenções a realizar na prevenção da PAVMI, faltando, em termos dos primeiros, a referenciação à avaliação diária do desmame ventilatório e/ou extubação, enquanto que em relação aos segundos, carece a colocação de uma SNG, a higienização das mãos e a necessidade de redução de antibioterapia profilática. Confirma-se assim que os resultados obtidos dos estudos selecionados corroboram as intervenções referidas na norma no 21/2012, de I6 de dezembro, com atualização a 30 de maio de 2017 (DGS, 2017b), embora 
os primeiros sejam mais atuais do que a norma existente, conseguindo obter-se mais intervenções direcionadas na prevenção da PAVMI.

Corrobora-se ainda a necessidade, sempre em conjunto ou "bundles", do planeamento, implementação, execução e avaliação das intervenções, como forma de potenciar o efeito das mesmas, reduzindo-se o seu efeito se as mesmas forem realizadas de forma isolada.

Já em relação às intervenções de enfermagem, as mesmas são especificadas nos estudos obtidos, referindo-se a autonomia completa do enfermeiro para o planeamento, implementação, execução e avaliação das mesmas, conseguindo ainda identificar-se duas intervenções que dependem de outros profissionais, como sendo a avaliação da sedação e a necessidade de redução de antibioterapia, enquanto que, no decorrer da norma no 21/2012, não existe especificação de classes profissionais.

\section{CONCLUSÕES}

A implementação de medidas preventivas, de forma precoce, se possível logo desde a instituição da via aérea artificial, vão permitir a efetividade na redução do risco de infeção, destacando-se nesta medida o papel fulcral do enfermeiro do Serviço de Urgência, responsável pelo planeamento, implementação, execução e avaliação das intervenções indispensáveis à prevenção e minimização dos efeitos tardios da PAVMI, habitualmente revelados após transferência dos doentes para outros serviços ou unidades hospitalares. Para além destas medidas preventivas, destaca-se ainda a necessidade de reforço de conhecimentos, teóricos, técnicos e práticos por parte de todos os intervenientes na prestação de cuidados diretos ao doente com VMI, para que se possa manter a qualidade e competência dos cuidados de saúde.

Para além do referido, assumem-se como contribuições para a prática clínica a necessidade de implementação de "bundles" ou conjunto de intervenções, no sentido de potenciar a prevenção da infeção, a realização destas intervenções apenas por profissionais com formação e conhecimentos adequados e a necessidade da manutenção das boas práticas, sem as quais não pode existir uma melhoria da prática clínica e um controlo de infeção eficaz. 
Como principal limitação desta revisão, pode-se apontar a quase inexistência de estudos de evidência científica relevantes, primeiro para o contexto português, e depois para a especificidade e dinâmicas existentes num serviço de urgência, já que a larga maioria dos artigos excluídos faziam referência a intervenções prestadas em contexto de cuidados intensivos, sugerindo-se, nesta medida, a realização de estudos sobre esta temática direcionados ao contexto de serviço de urgência.

\section{REFERÊNCIAS BIBLIOGRÁFICAS}

American Psychological Association (2020). Publication manual of the American Psychological Association, $7^{\text {th }}$ ed. Washington: APA. ISBN: 978143383216r;

Aromataris, E. \& Munn, Z. (2020). JBI Manual for Evidence Synthesis. DOI: 10.46658/jbimes-20-or. ISBN: 978-o-6488488-o-6;

Decreto-Lei no 16I/96, de 4 de setembro, do Ministério da Saúde (1996). Aprova o Regulamento do Exercício Profissional dos Enfermeiros. Diário da República, I Série-A, no 205, 2959-2962. Disponível em https://bit.ly/2T74w Ti;

Direção-Geral da Saúde (2017a). Programa de Prevenção e Controlo de Infeções e de Resistência aos Antimicrobianos. Lisboa: DGS. Disponível em https://bit.ly/3qngnbC;

Direção-Geral da Saúde (2017b). Norma no 21/2015 - "Feixe de Intervenções" de Prevenção de Pneumonia Associada à Intubação. Disponível em https://bit.ly/2SolrQw;

Ferreira, E., Kimura, A., Ramos,D., Albuquerque, P., Antunes, M. \& Oliveira, D. (2017). Prevalência de pneumonia associada à ventilação mecânica por meio de análise das secreções traqueobrônquicas. Revista da Rede de Enfermagem do Nordeste, I8(I), eo46o. DOI: I0.15253/2175-6783.2017000100016;

Frota, M., Campanharo, C., Lopes, M., Piacezzi, L., Okuno, M. \& Batista, R. (2019). Good practices for preventing ventilator-associated pneumonia in the emergency department. Revista da Escola de Enfermagem da USP, 53, e046o. DOI: 10.1590/Sig80-220X2018010803460;

Jameson, J., Kasper, D., Fauci, A. Hauser, S., Longo, D. \& Loscalzo, J. (2015). Harrison's principles of internal medicine. New York: McGraw-Hill Education. ISBN: 978-0-07-180216-I;

Lito, L. (2010). Epidemiologia da infeção hospitalar. Cadernos de Saúde, 3(especial), 25-31. DOI: 10.34632/cadernosdesaude.2010.2988;

Lobão, M., Sousa, P., Almeida, G. \& Alves, J., Mendes, J., Perelman, J. \& Campos, A. (2016). Infecções Associadas aos Cuidados de Saúde: Contributo da indústria de meios de diagnóstico in vitro para o seu controlo. Disponível em https://bit.ly/3h5 KekD; 
Maran, E., Melo, W., Spigolon, D., Teston, E., Tostes, M. \& Barreto, M. (2019). Prevention of pneumonia associated with mechanical ventilation under the optics of nursing academics / Prevenção da pneumonia associada à ventilação mecânica sob a ótica de acadêmicos de enfermagem. Revista De Pesquisa Cuidado é Fundamental Online, II(I), II8I23. DOI: 10.9789/2175-5361.2019.viriI.II8-I23;

Ministério da Saúde (2018). Retrato da Saúde. Portugal: Ministério da Saúde. Disponível em https://bit.ly/3jfDZgX;

Oliveira, A., Rocha, A., Silva, C., Matos, D., Lima, E., Anjos, E., Silva, F., Silva, I., Mendes, J., Nogueira, P., Silva, R., Luz, S., Lopes. T., Guimarães, V., Silva, W. \& Nascimento, W. (2020). Pneumonia Associada à Ventilação Mecânica: O Cuidar da Enfermagem. Brazilian Journal of Surgery and Clinical Research, 29(3), 37-4I. Disponível em https://bit.ly/36oqWYU. ISSN: 2317-4404;

Ordem dos Enfermeiros (2017). Padrões de qualidade dos cuidados especializados em enfermagem médico-cirúrgica. Assembleia Extraordinária do Colégio da Especialidade de Enfermagem MédicoCirúrgica. Leiria: Ordem dos Enfermeiros. Disponível em https://bit.ly/2MGBUNj;

Pina, E., Ferreira, E. \& Marques, A. (2010). Infeções associadas aos cuidados de saúde e segurança do doente. Revista Portuguesa de Saúde Pública, Io, 27-39. Disponível em https://bit.ly/35TC8q6;

Reis, A. (2017). Prevenção da Pneumonia Associada à Ventilação na Pessoa em Situação Crítica Intervenção Especializada de Enfermagem (Relatório de Estágio, Escola Superior de Enfermagem de Lisboa). Disponível em https://bit.ly/368zugz;

Rodrigues, J. (2019). Impacto económico e clínico da Infeção da Corrente Sanguínea relacionada com o Cateter Venoso Central e da Pneumonia associada à Ventilação em Unidades de Cuidados Intensivos de um Centro Hospitalar Universitário (Dissertação de Mestrado em Gestão da Saúde, Escola Nacional de Saúde Pública da Universidade Nova de Lisboa). Disponível em https://bit.ly/2SMzINN;

Santos, W., Secoli, S. \& Puschel, V. (2018). A abordagem do Joanna Briggs Institute para revisões sistemáticas. Revista Latino-Americana de Enfermagem, 26(o). DOI: 10.1590/15188345.2885.3074;

Simões, C. (2016). Infeções Hospitalares Bacterianas no Século XXI (Tese de Mestrado em Ciências Farmacêuticas, Faculdade de Ciências da Saúde da Universidade Fernando Pessoa). Disponível em https://bit.ly/3x5H8ny;

Xu, Y., Lai, C., Xu, G., Meng, W., Zhang, J., Hou, H. \& Pi, H. (2019). Risk factors of ventilator-associated pneumonia in elderly patients receiving mechanical ventilation. Clinical Interventions in Aging, 14, 1027-1038. DOI: 10.2147/cia.s197146. 